\title{
Solar impulsive soft $X$-ray brightenings and their connection with footpoint hard $X$-ray emission sources
}

\author{
T. Mrozek and M. Tomczak
}

\author{
Astronomical Institute, University of Wrocław, ul. Kopernika 11, 51-622 Wrocław, Poland \\ Received 25 April 2003 / Accepted 5 September 2003
}

\begin{abstract}
We have investigated 46 solar flares showing impulsive soft X-ray brightenings in footpoints. To obtain their main observational characteristics we used the Yohkoh/SXT images. The results are presented in the form of normalized histograms showing the distribution of the characteristics. For 18 flares it has been possible to make a quantitative comparison between the footpoint response in soft X-ray images and in hard X-ray images. The latter have been derived from the Yohkoh/HXT and reconstructed using the MEM procedure. The evident correlation between these two kinds of response, as well as between the deposited energies, strongly supports a common physical cause, namely non-thermal electron beams. We present evidence that in the impulsive phase the chromospheric evaporation is driven mainly by low-energy non-thermal electrons. We show that the low-energy cut-off energy in hard X-ray photon fluxes may vary from flare to flare and may even vary during a given flare.
\end{abstract}

Key words. Sun: corona - flares - X-rays, gamma rays

\section{Introduction}

A relationship between hard X-ray radiation (HXR) and soft $\mathrm{X}$-ray radiation (SXR) of solar flares has been discussed from many aspects. Very well known is a similarity between the time derivative of the SXR flux and the HXR flux called the Neupert effect. Several statistical studies of the Neupert effect for a large number of solar flares considering total HXR and SXR light curves have been performed (Dennis \& Zarro 1993; Lee et al. 1995; McTiernan et al. 1999; Veronig et al. 2002). The main conclusion of them was that the Neupert effect is evidence of chromospheric evaporation driven by non-thermal electron beams.

The Japanese satellite Yohkoh offered a possibility to investigate the precipitation of non-thermal electrons in solar flares in a more comprehensive way. Comparing images made during the impulsive phase of flares in HXRs and in SXRs, we can study such a process operating in flare footpoints in two ways. First, we should see HXR bremsstrahlung of non-thermal electrons which are stopped at the entrance of magnetic coronal structures into the denser part of the solar atmosphere. This mechanism, known as the thick-target model (Brown 1971), is responsible for the footpoint HXR emission sources seen in HXR images (Sakao 1994). Second, a response is expected of the environmental plasma due to incoming energy deposited by non-thermal electrons. This response in SXR images takes the form of a radiation enhancement called the impulsive SXR brightening (Hudson et al. 1994).

Send offprint requests to: T. Mrozek, e-mail: mrozek@astro.uni.wroc.pl
Using the imaging capabilities of Yohkoh, Tomczak (1999) made the first attempt to study the spatial resolution of the Neupert effect. He showed that impulsive SXR brightenings are directly responsible for the Neupert effect. He also made a quantitative comparison between the HXR and SXR reaction to non-thermal electrons in flare footpoints and concluded that impulsive SXR brightenings are mainly produced by nonthermal electrons with relatively low energies.

The results of Tomczak (1999) are based on a sample of only five flares. Therefore, in this paper we repeat his analysis for a larger number of events to check if his results depend on selection effects. The paper is organized in the following way. Section 2 contains the description of the observing instruments and characterizes a set of selected events. In Sect. 3 the analysis techniques of the data are presented. The main results are presented in Sect. 4, including histograms of basic observational characteristics of impulsive SXR brightenings. The results are summarized in Sect. 5.

\section{Observations}

In our analysis we used data from two imaging instruments installed on board the Japanese satellite Yohkoh (Ogawara et al. 1991). The Soft X-ray Telescope, SXT (Tsuneta et al. 1991), is a grazing-incidence telescope equipped with a $1024 \times$ $1024 \mathrm{CCD}$ and a set of filters covering the spectral range from 3 to $45 \AA$. The SXT observed the whole Sun, but during the flare mode only a part of the CCD was read out, namely the part where the SXT recorded the strongest signal. Such partial frames have a typical size of $64 \times 64$ pixels and a spatial resolution of 2 '. $45 \times 2$ '. 45 . The time resolution is determined by 
the time interval between two exposures with the same filter (typically 2-14 s).

The Hard X-ray Telescope, HXT (Kosugi et al. 1991), is a Fourier synthesis imager observing the whole Sun. It consists of 64 independent subcollimators which measure spatially modulated intensities in four energy bands from 14 to $93 \mathrm{keV}$ (L: 14-23 keV, M1: 23-33 keV, M2: 33-53 keV and H: 53$93 \mathrm{keV}$ ). During the flare the intensities were integrated, in each energy band, over $0.5 \mathrm{~s}$ and this is the maximum time resolution for HXR light curves. Using various reconstruction methods, e.g. Maximum Entropy Method, MEM, (Sakao 1994) or pixons (Metcalf et al. 1996), we can obtain HXR images with angular resolutions up to $5^{\prime \prime}$. The time resolution for HXR images depends on the counting rate and varies typically from 0.5 to several tens of seconds.

There were some flares for which HXR data were not available from the HXT. For these, we used data from the Burst and Transient Source Experiment (BATSE) on board the Compton Gamma Ray Observatory (CGRO). This instrument was more sensitive than the HXT, thus it allowed us to investigate also the relatively weak flares. Due to a lack of imaging capabilities of the BATSE, we could use its data only to determine temporal characteristics of flares.

In summary, we analyzed 46 flares (see Table 1) with the well-observed impulsive phase showing impulsive SXR brightenings which have been found during a prompt inspection of the SXT images. They cover longitudes from E90 to W90 and GOES classes from $\mathrm{C} 2.7$ to X1.2. The wide range of longitudes and classes enables us to investigate general characteristics of impulsive SXR brightenings, which do not depend on flare position or intensity.

\section{Analysis}

For each flare in Table 1 we chose a time interval from the start of the impulsive phase to about 2 min after its end. The set of images covering this interval has been processed using the standard SXT_PREP routine (Morrison 1994). We used the SXT images made with the Al12 filter because in this filter impulsive SXR brightenings are the most spectacular. An exception are events Nos. 19-21, 28 and 40, for which only the SXT images made with the Be119 filter were available.

In the first image of a sequence we determined the area of the flare including all pixels that show a signal above $10 \%$ of the brightest pixel. In the next step, we analyzed the light curve of each unsaturated pixel from the selected area. We defined an impulsive SXR brightening as a change of brightness which showed a rise to a maximum value with a following drop and took place not later than $2 \mathrm{~min}$ after the end of the impulsive phase.

To obtain more information on the behaviour of such a brightening we compared the light curve for each investigated pixel to its time-smoothed equivalent. We calculated the running mean involving 3-7 time points depending on the time resolution. Having both time-smoothed and observed values available, we were able to obtain the standard deviation $(\sigma)$ for the light curve of each pixel. Next, for each point of the light curve, we calculated the difference between observed and

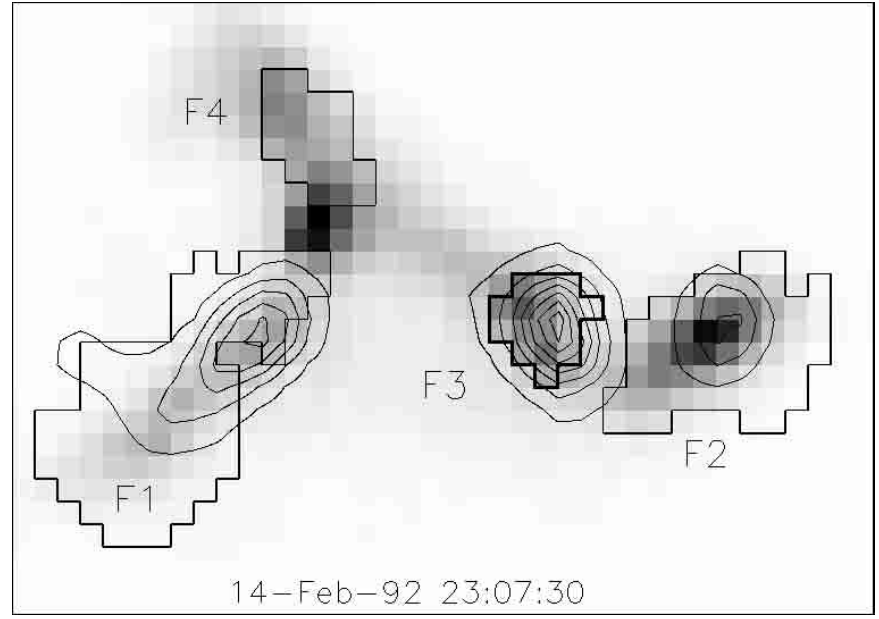

Fig. 1. Image of the 14 February 1992 flare obtained at the maximum of the HXR light curve. The grey scale represents the SXT(A112) emission distribution. The regions of the SXR response are marked F1, F2, F3 and F4, respectively. The contours of the HXR emission are overplotted $(95,80,65,50,35,20$ and $5 \%$ of the maximum intensity).

time-smoothed values. If this difference exceeded $0.8 \sigma$ and for neighbouring points of the light curve exceeded $0.3 \sigma$, then we took such an increase of brightness under consideration and calculated the total signal for it. If the total signal exceeded the time smoothed value by $2 \sigma$ then we concluded that we found a case of impulsive SXR brightening.

The threshold values $(0.8 \sigma$ and $0.3 \sigma)$ that we used can vary from flare to flare; therefore for each event we had to verify manually the results obtained from our program. We excluded sudden signal jumps in individual pixels caused by statistical noise, cosmic rays, telemetry errors etc.

We assumed that neighbouring pixels showing similar time profiles of impulsive SXR brightenings responded to a common non-thermal electron beam. We call such a set of pixels the region of the SXR response. The actual sizes of such regions were somewhat broadened due to the Point Spread Function of the SXT (Martens et al. 1995). For each flare we found several regions of the SXR response (see Table 1, Col. 9) and localized them at footpoints of the flaring magnetic structures (see Figs. 1 and 3). Sometimes one region of the SXR response showed several impulsive brightenings. Altogether, 182 regions showing 228 impulsive SXR brightenings were found for the investigated flares. The number of identified footpoints per flare varies from 1 to 11 .

Apart from the impulsive component caused by nonthermal electron beams, the SXR light curves also contain a gradual, slowly-varying component. We presumed that this is a manifestation of the thermal conduction as well as a result of instrumental factors and assumed a linear rise in time during the impulsive phase (Tomczak 1999). After subtracting the gradual component we obtained a net signal, describing a pure impulsive SXR response due to non-thermal electrons. Examples of the total SXT signal before subtraction and of the net signal can be seen in Figs. 2 and 4. 
Table 1. List of investigated flares.

\begin{tabular}{|c|c|c|c|c|c|c|c|c|c|c|c|}
\hline (1) & (2) & (3) & (4) & (5) & (6) & (7) & (8) & (9) & (10) & (11) & (12) \\
\hline 1 & $24-O C T .-91$ & $22: 38$ & $22: 41$ & $M 9.8$ & $1 N$ & $S 12 E 46$ & 6891 & 4 & 6 & & + \\
\hline 2 & $02-N O V .-91$ & $06: 47$ & $06: 53$ & $M 9.0$ & $1 B$ & $S 13 W 61$ & 6891 & 4 & 5 & $130-150$ & + \\
\hline 3 & $09-N O V .-91$ & $03: 13$ & 03:20 & $M 1.5$ & $S N$ & $N 20 E 08$ & 6918 & 2 & 2 & & - \\
\hline 4 & $10-N O V .-91$ & $20: 10$ & $20: 1$ & $M 7.9$ & $1 N$ & $S 15 E 43$ & 6919 & 3 & 3 & & + \\
\hline 5 & $19-N O V .-91$ & $09: 29$ & $09: 32$ & $C 8.5$ & $1 F$ & $S 12 W 60$ & 6919 & 5 & 5 & $280-560$ & + \\
\hline 6 & $13-J A N .-92$ & $17: 29$ & $17: 34$ & $M 2.0$ & - & $S 15 W 90+$ & 6994 & 2 & 2 & $260-310$ & + \\
\hline 7 & $26-J A N .-92$ & $15: 28$ & $15: 33$ & $X 1.0$ & $3 B$ & $S 16 W 66$ & 7012 & 6 & 7 & $360-640$ & + \\
\hline 8 & $14-F E B .-92$ & 23:07 & $23: 10$ & $M 7.0$ & $2 B$ & $S 13 E 02$ & 7056 & 4 & 4 & $220-380$ & + \\
\hline 9 & $17-F E B .-92$ & $15: 42$ & $15: 46$ & $M 1.9$ & $S F$ & $N 16 W 81$ & 7050 & 3 & 5 & & + \\
\hline 10 & $28-J U N .-92$ & $13: 57$ & & $M 1.6$ & - & $N 15 E 90+$ & 7216 & 7 & 14 & $400-720$ & - \\
\hline 11 & $08-J U L .-92$ & $09: 4$ & $09: 5$ & $X 1.2$ & $1 B$ & $S 11 E 46$ & 7220 & 4 & 8 & $280-450$ & + \\
\hline 12 & $15-J U L .-92$ & 02:00 & 02: & $C 4.2$ & - & & 7222 & 4 & 6 & & + \\
\hline 13 & $11-A U G .-92$ & 13: & & & $1 N$ & & 7248 & 3 & 3 & & + \\
\hline 14 & $11-A U G .-92$ & $22: 25$ & 22: & $M 1.4$ & - & $N 15 E 90+$ & 7260 & 5 & 5 & $380-560$ & - \\
\hline 15 & $06-S E P .-92$ & $05: 16$ & & $M 2.4$ & $2 N$ & $S 09 W 39$ & 7270 & 3 & 5 & & - \\
\hline 16 & $06-S E P .-92$ & 09:04 & 09: & M3.3 & $1 N$ & $S 11 W 38$ & 7270 & 3 & 3 & & + \\
\hline 17 & $11-S E P .-92$ & 03:0 & 03: & $M 1$ & - & $N 17 E 40$ & 7276 & 3 & 5 & & + \\
\hline 18 & $12-S E P .-92$ & $15: 39$ & 15: & $C 5$ & $S N$ & $N 18 E 21$ & 7276 & 2 & 2 & & + \\
\hline 19 & $02-F E B-93$ & $03: 46$ & 03: & $C 6.3$ & $1 N$ & $S 16 E 13$ & 7412 & 3 & 4 & & - \\
\hline 20 & $03-F E B .-93$ & $14: 59$ & $15:($ & C8.8 & $1 F$ & $N 10 E 64$ & 7417 & 2 & 2 & & - \\
\hline 21 & $06-F E B .-93$ & $20: 4$ & 2 & $C 7.5$ & $S F$ & $S 08 E 54$ & 7420 & 2 & 2 & & - \\
\hline 22 & $02-M A R .-93$ & 15: & & & $S F$ & $S 07 E 82$ & 7440 & 2 & 2 & 240 & - \\
\hline 23 & $27-S E P .-93$ & $10: 52$ & $10: 5$ & C5.7 & $S F$ & $N 11 E 80$ & 7590 & 3 & 5 & & - \\
\hline 24 & $30-N O V .-93$ & 06:04 & $06: 0$ & $C 9.2$ & - & $S 20 E 90+$ & 7627 & 5 & 5 & $580-630$ & - \\
\hline 25 & $16-J A N .-94$ & $23: 20$ & $23: 2$ & M6.1 & $1 B$ & N09E73 & 7654 & 3 & 5 & & + \\
\hline 26 & $30-J U N .-94$ & $21: 21$ & $21: 2$ & $M 2$ & $1 B$ & $12 E 27$ & 7742 & 2 & 3 & & - \\
\hline 27 & $13-$ OCT. -95 & & & & $1 F$ & & 7912 & 8 & 11 & & - \\
\hline 28 & $25-N O V .-96$ & $00: 16$ & $00: 19$ & $C 8.0$ & $1 F$ & $3 E 16$ & 7999 & 1 & 1 & & - \\
\hline 29 & $29-A U G .-97$ & $23: 31$ & $23: 3$ & $M 1.4$ & $S F$ & $N 31 E 16$ & 8076 & 6 & 6 & & - \\
\hline 30 & $02-S E P .-97$ & $21: 11$ & $21: 1$ & $C 4.1$ & $S F$ & $N 32 W 41$ & 8076 & 2 & 2 & & + \\
\hline 31 & $14-S E P .-97$ & $02: 53$ & & & $S F$ & & 8083 & 3 & 3 & & + \\
\hline 32 & $22-S E P .-97$ & $18: 10$ & $18: 17$ & $C 5.1$ & $S F$ & $S 28 E 41$ & 8088 & 2 & 3 & & - \\
\hline 33 & $05-N O V .-97$ & $10: 04$ & $10: 06$ & $C 8.1$ & - & $S 20 W 45$ & 8100 & 5 & 5 & & - \\
\hline 34 & $14-N O V .-97$ & $10: 32$ & $10: 38$ & $C 4.6$ & $S F$ & $N 21 E 70$ & 8108 & 4 & 4 & & - \\
\hline 35 & $17-N O V .-97$ & $15: 0$ & & & $S F$ & & 8108 & 3 & 7 & & - \\
\hline 36 & $01-D E C .-97$ & $02: 35$ & & $M 1.2$ & $1 N$ & $N 20 E 24$ & 8113 & 3 & 5 & & - \\
\hline 37 & $27-D E C .-97$ & $16: 33$ & $16: 38$ & $C 3.2$ & $1 F$ & $S 23 W 06$ & 8124 & 7 & 7 & & - \\
\hline 38 & $15-J A N .-98$ & 09:30 & 09:3 & C6.8 & $S F$ & $S 24 W 29$ & 8131 & 5 & 6 & & - \\
\hline 39 & $15-J A N .-98$ & $14: 34$ & 14: & $M 1.0$ & $1 F$ & $S 22 W 30$ & 8131 & 4 & 5 & & - \\
\hline 40 & $04-M A R .-98$ & $21: 33$ & & $C 2.7$ & $S F$ & $S 22 W 59$ & 8171 & 2 & 2 & & - \\
\hline 41 & $05-M A R .-98$ & $12: 34$ & $12: 37$ & $C 2.6$ & - & $S 21 W 67$ & 8171 & 4 & 4 & & - \\
\hline 42 & $01-M A Y-98$ & $12: 58$ & $13: 00$ & $M 1.1$ & $1 N$ & $N 25 E 40$ & 8214 & 6 & 7 & & - \\
\hline 43 & $02-M A Y-98$ & $04: 57$ & 05:00 & $C 5.4$ & - & $S 19 W 08$ & 8210 & 5 & 5 & & - \\
\hline 44 & $28-M A Y-98$ & $19: 05$ & $19: 12$ & $C 8.7$ & $S F$ & S 20W07 & 8210 & 11 & 14 & & + \\
\hline 45 & $26-N O V .-99$ & $13: 43$ & $13: 43$ & M6.0 & $2 N$ & $S 15 W 59$ & 8771 & 7 & 7 & & - \\
\hline 46 & $27-N O V .-99$ & $05: 47$ & $05: 49$ & $M 1.2$ & $1 N$ & $S 14 W 63$ & 8771 & 5 & 5 & & - \\
\hline
\end{tabular}

(1) Number of event; (2) - date; (3) - HXR maximum time [UT]; (4) - GOES maximum time [UT]; (5) - GOES class; (6) - H $\alpha$ importance; (7) - location; (8) - NOAA AR; (9) - number of regions of the SXR response; (10) - number of impulsive SXR brightening; (11) - range of estimated evaporation velocities $\left[\mathrm{km} \mathrm{s}^{-1}\right] ;(12)-(+)$ means that the quantitative analysis of the HXR images has been done.

To verify the source of the gradual component we compared the estimated conductive flux in the flare footpoints to values of the signal taken from the linear approximation (dotted lines in Figs. 2 and 4). This was done for the time of the maximum of net light curves. The classical formula of conductivity for a fully ionizated hydrogen plasma was used
(Spitzer 1962). We made the comparison only for the flares located near the solar limb, to limit the loop-length uncertainty. The comparison presented in Fig. 5 shows the good correlation $(R=0.40)$ between the conductive flux and the gradual component which supports our interpretation. 

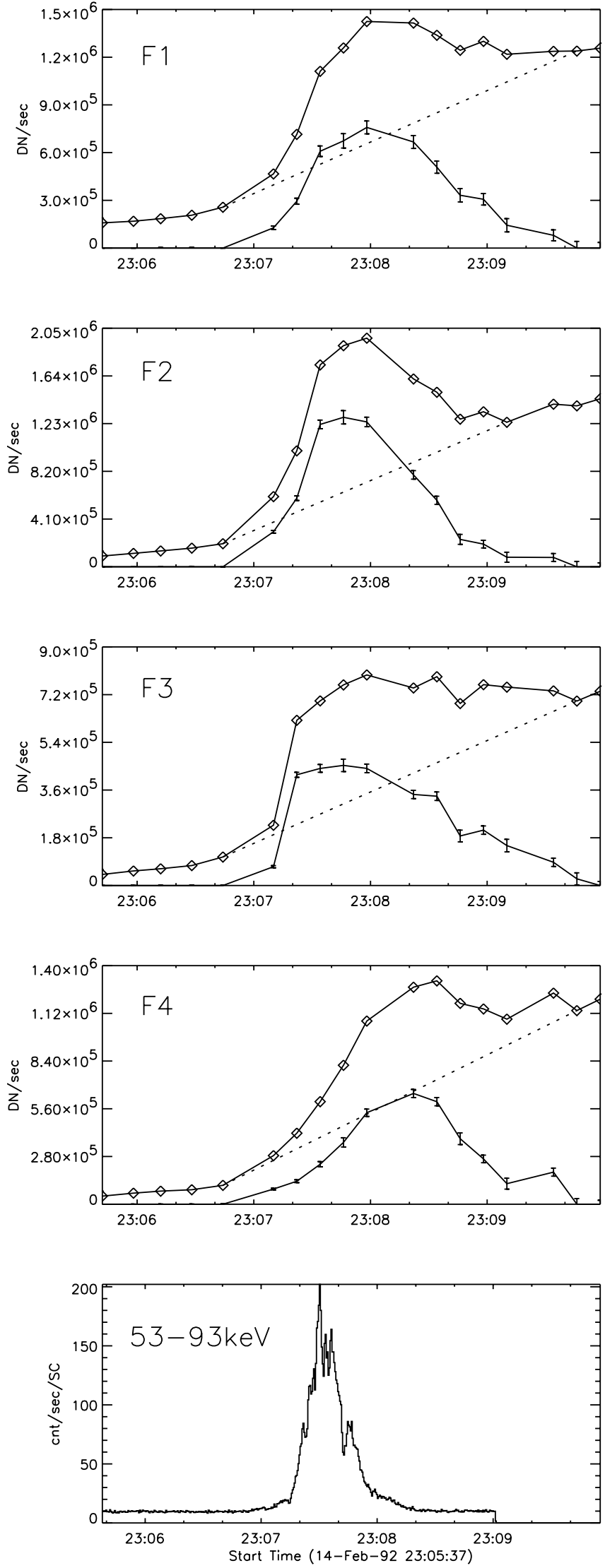

Fig. 2. The SXT(A112) light curves for the footpoints marked in Fig. 1. The total as well as the net light curves are presented. The subtracted gradual component is marked by a dotted line. In addition, the total $\mathrm{HTX}(\mathrm{H})$ light curve is shown.
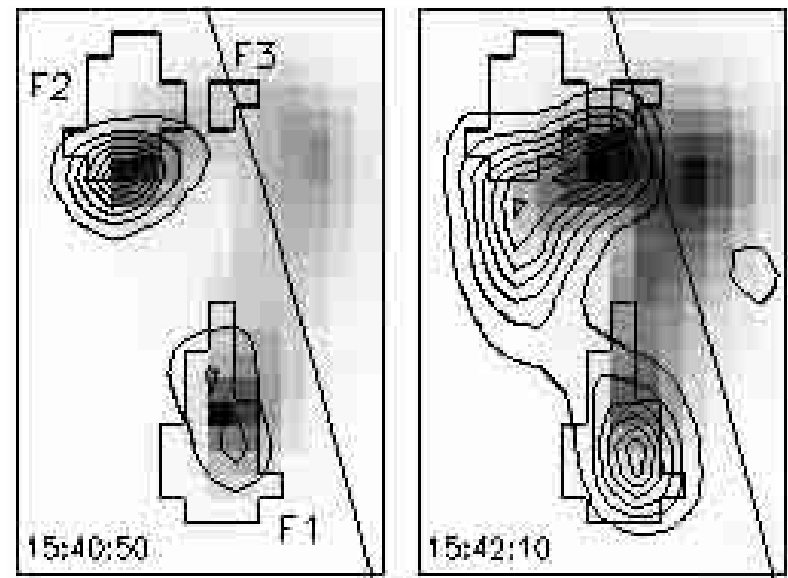

Fig. 3. Two images of the 17 February 1992 flare obtained at maxima of the HXR light curve. The grey scale represents the SXT(Al12) emission distribution. The regions of the SXR response are marked F1, F2 and F3, respectively. The contours of the HXR emission are overplotted $(95,80,65,50,35,20$ and $5 \%$ of the maximum intensity).

A majority of the regions of the SXR response found was associated with footpoint HXR emission sources. We obtained HXR images using the standard HXT_MULTIMAGE routine. Considering the large number of events we chose the Maximum Entropy Method (Sakao 1994), which works relatively fast and gives images of good quality, especially after including new modulation patterns computed by Sato et al. (1999).

The relative positions of impulsive SXR brightenings and footpoint HXR emission sources can be seen in Figs. 1 and 3 showing the 14 February 1992 and the 17 February 1992 flares, respectively. A good spatial correlation can be seen as well as agreement in shapes. The expected correlation between the sizes of regions of the SXR response and the footpoint HXR emission sources is strongly deformed by instrumental effects like the PSF of the SXT and the convergence of reconstruction methods of HXR images. In some cases we observed SXR footpoints without visible HXR emission (for example F4 in Fig. 1) which we interpret as an effect of the low dynamic range of the HXT instrument (Sakao 1994).

We estimated the area of footpoint HXR emission sources as follows. For each channel we made a set of images covering the time interval near the maximum of the HXR burst. We carefully determined the borders of each reliable HXR emission source, common for all channels, using all available images. As "reliable" we treated the sources within the dynamical range of the HXT (brighter than $10 \%$ of the maximum intensity) which were present in the whole set of images. Further, we used the images obtained at the maximum of the main HXR bursts and estimated the brightness of such sources adding the whole signal within the determined borders for each channel.

\section{Results}

\subsection{Characteristics of impulsive SXR brightenings}

We have defined the following characteristics of impulsive SXR brightenings:

1. FWHM duration time, $t,-$ a time interval during which the signal was above half the maximum value, 

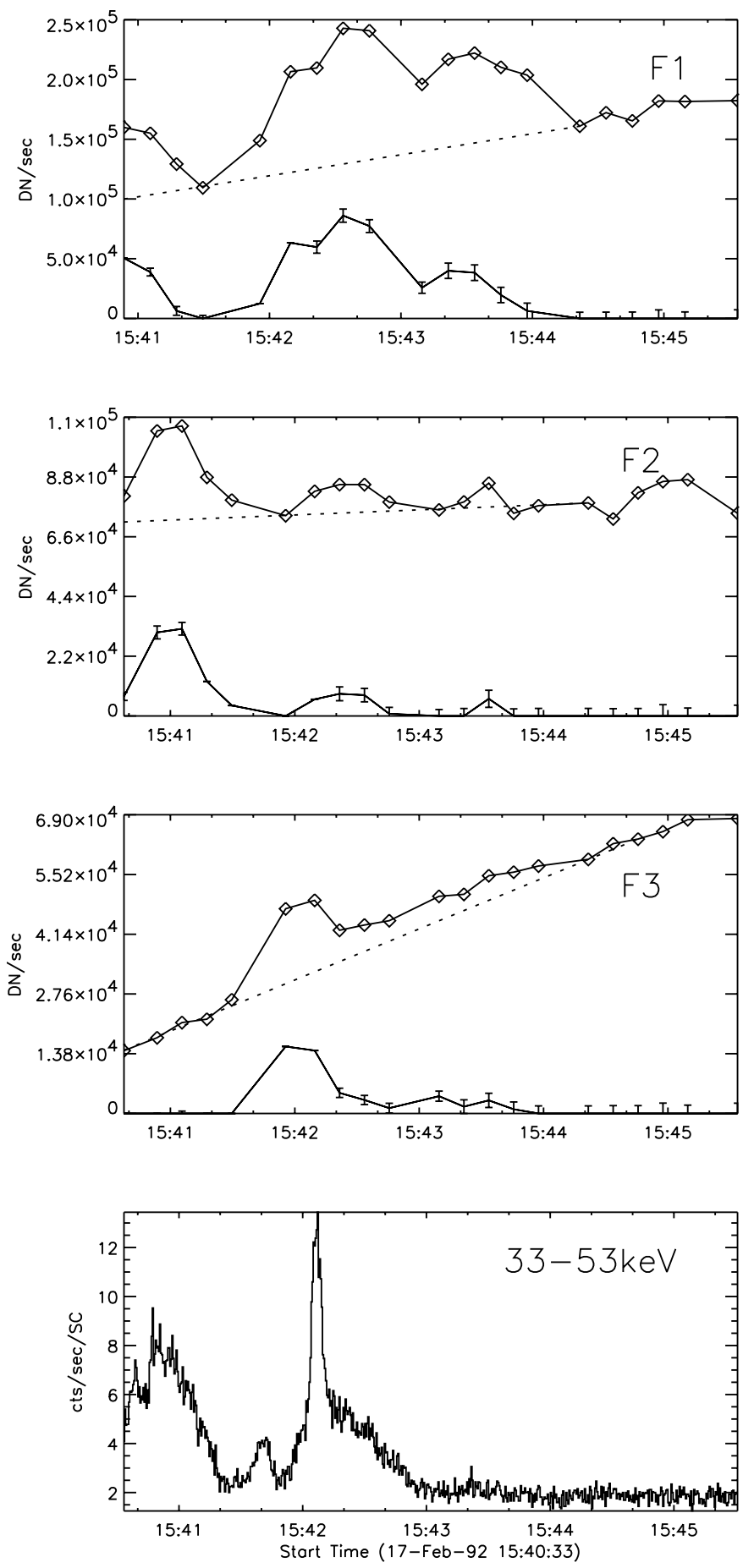

Fig. 4. The SXT(A112) light curves for the footpoints marked in Fig. 3. The total as well as the net light curves are presented. The subtracted gradual component is marked by a dotted line. In addition, the total $\operatorname{HTX}(\mathrm{M} 2)$ light curve is shown.

2.-3. time profile characteristics measured by the time interval ratios: $t_{\mathrm{r}} / t$, and $t_{\mathrm{d}} / t$, where $t_{\mathrm{r}}$, and $t_{\mathrm{d}}$ are the time intervals during which the signal was above half the maximum value, during phases of the rise and decay, respectively,

4. relative brightness $I_{\mathrm{F}} / I_{\text {tot }}$ - the part of the SXR emission of the flare which was due to non-thermal electron heating in a particular footpoint,

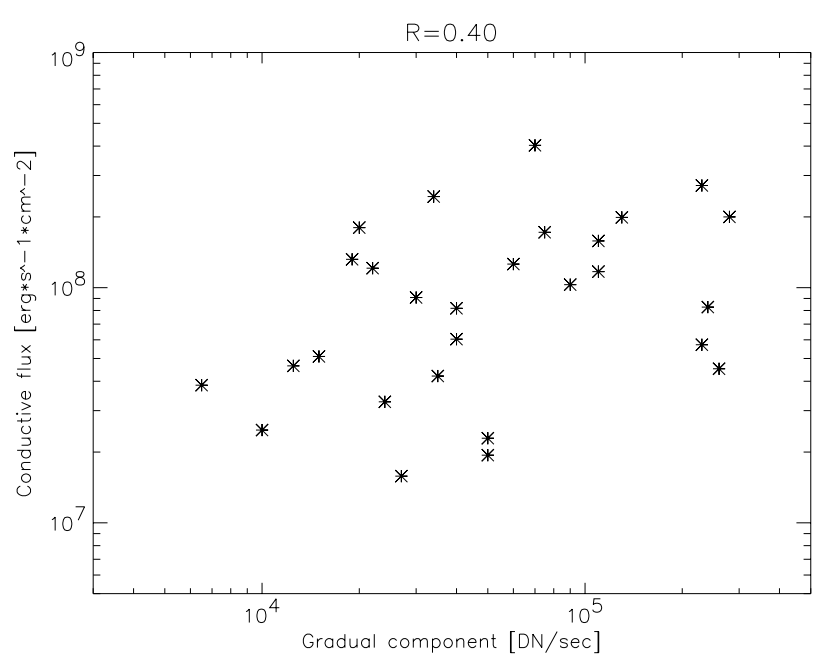

Fig. 5. Relation between the conductive flux calculated for near-thelimb events and the flux obtained from the linear approximation of the gradual component. $R$ is the correlation coefficient.

5. SXR/HXR delay - the time difference between the maxima of the net SXR light curve and the HXR light curve,

6. relative duration of the response to the non-thermal electrons in the SXR and the HXR radiation, $t / t_{\mathrm{HXR}}$, where $t_{\mathrm{HXR}}$ is the FWHM duration in the HXR radiation,

7.-8. temperature, $T$, and electron density, $n_{\mathrm{e}}$, of an SXR footpoint brightening,

9. FWHM area of an SXR brightening, $S$, - number of SXT pixels having a signal above $50 \%$ of the brightest pixel in this footpoint.

We used the net SXR light curves to obtain characteristics (1)(4) as well as net SXR light curves and total HXR light curves to obtain characteristics (5)-(6). Temperatures $T$ and emission measures $(\varepsilon / V)$ have been obtained from the signal ratio in the Be119 and Al12 images according to the signal ratio method (Hara et al. 1992). We derived the average values over the FWHM area, $S$, of the footpoints. For this calculation we used images corresponding to the time of the maximum of the net light curves. The electron density, $n_{\mathrm{e}}$ was calculated from the formula $n_{\mathrm{e}}=(\varepsilon / V)^{0.5}$, where the volume $V$ is the product of the FWHM area, $S$ and the thickness which we assumed to be equal to the smaller diameter of the FWHM area. We used the FWHM area of footpoints instead of the whole area of regions of the SXR response to limit the influence of the PSF of the SXT on our results.

For each of 228 impulsive SXR brightenings characteristics (1)-(9) have been obtained and the results are presented in Figs. $6-8$ in the form of normalized histograms (i.e. the total value of all bins is always equal to 1 ). Each panel also contains information about the number of events and the size of the bins. The bin size was taken to be approximately equal to the uncertainties of the values displayed on the $x$-axis of the histogram. In the histograms of temperature and electron density, the number of events is lower as there were only single-filter observations available for flares Nos. 19-21, 28 and 40.

In the histogram of the FWHM duration (Fig. 6, left panel) more than $70 \%$ of events falls into the interval of $20-70 \mathrm{~s}$. 

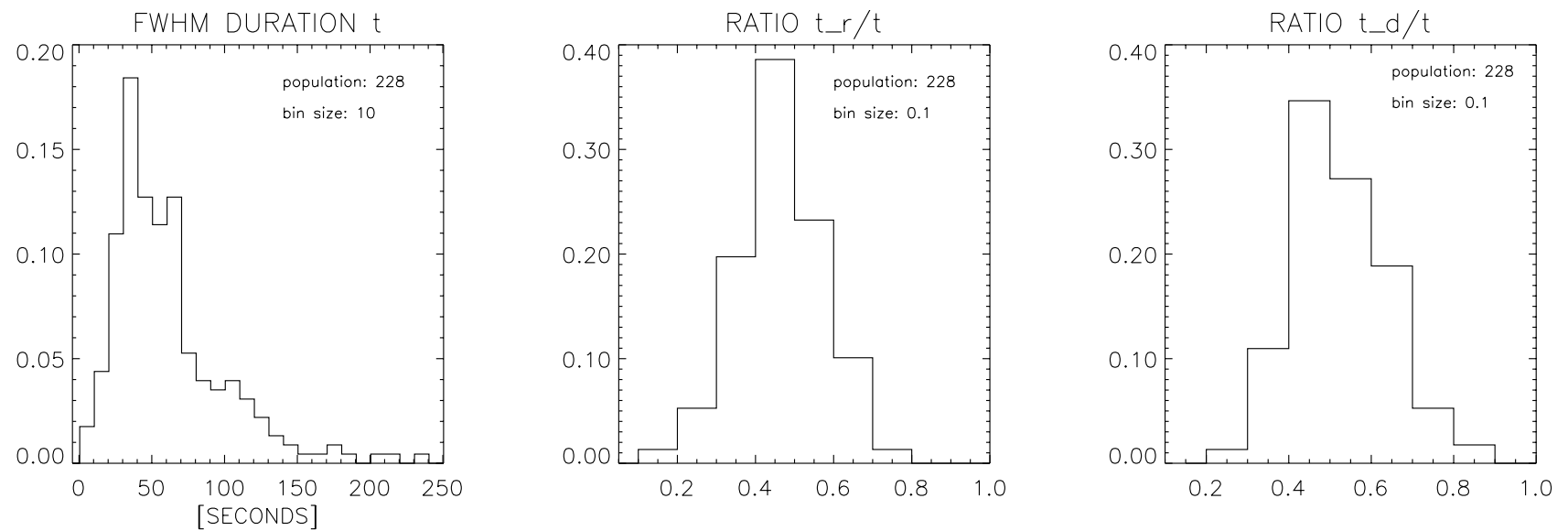

Fig. 6. Normalized histograms of the FWHM duration $t$, the ratio $t_{\mathrm{r}} / t$, and the ratio $t_{\mathrm{d}} / t$ (left, middle, and right panel, respectively) for investigated impulsive SXR brightenings.
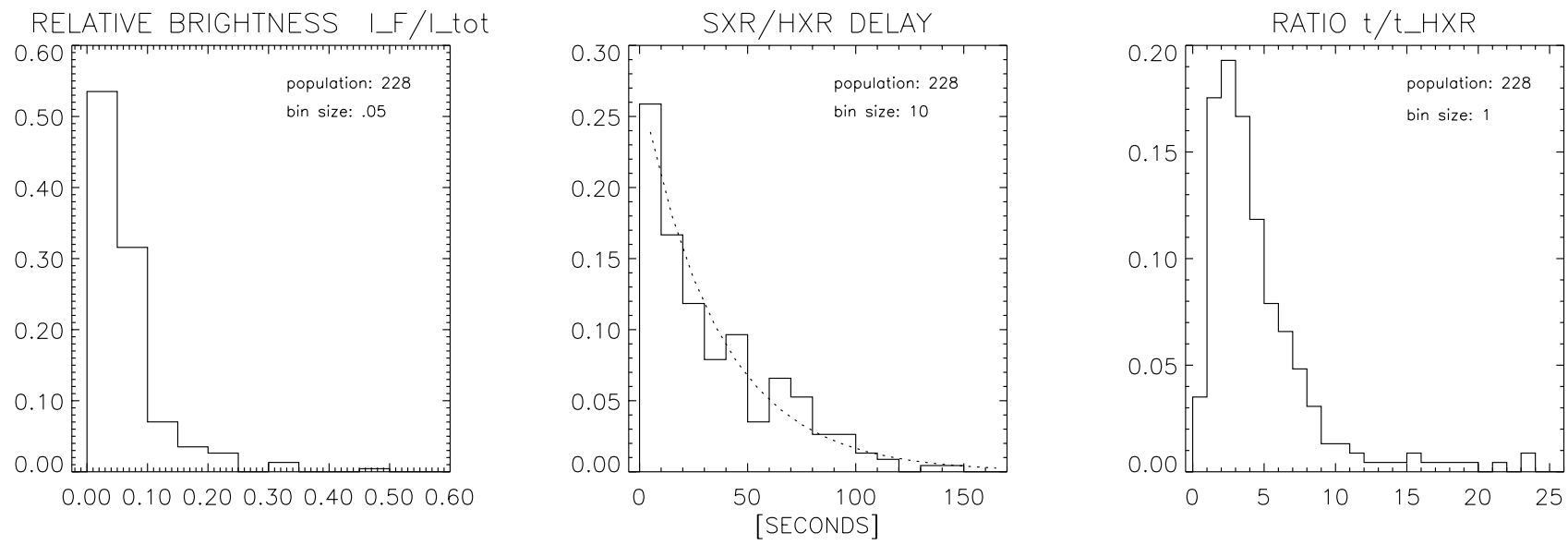

Fig. 7. Normalized histograms of the ratio $I_{\mathrm{F}} / I_{\mathrm{tot}}$, the SXR/HXR delay, and the ratio $t / t_{\mathrm{HXR}}$ (left, middle, and right panel, respectively) for investigated impulsive SXR brightenings. In the middle panel the Poisson distribution is fitted (the dotted line).
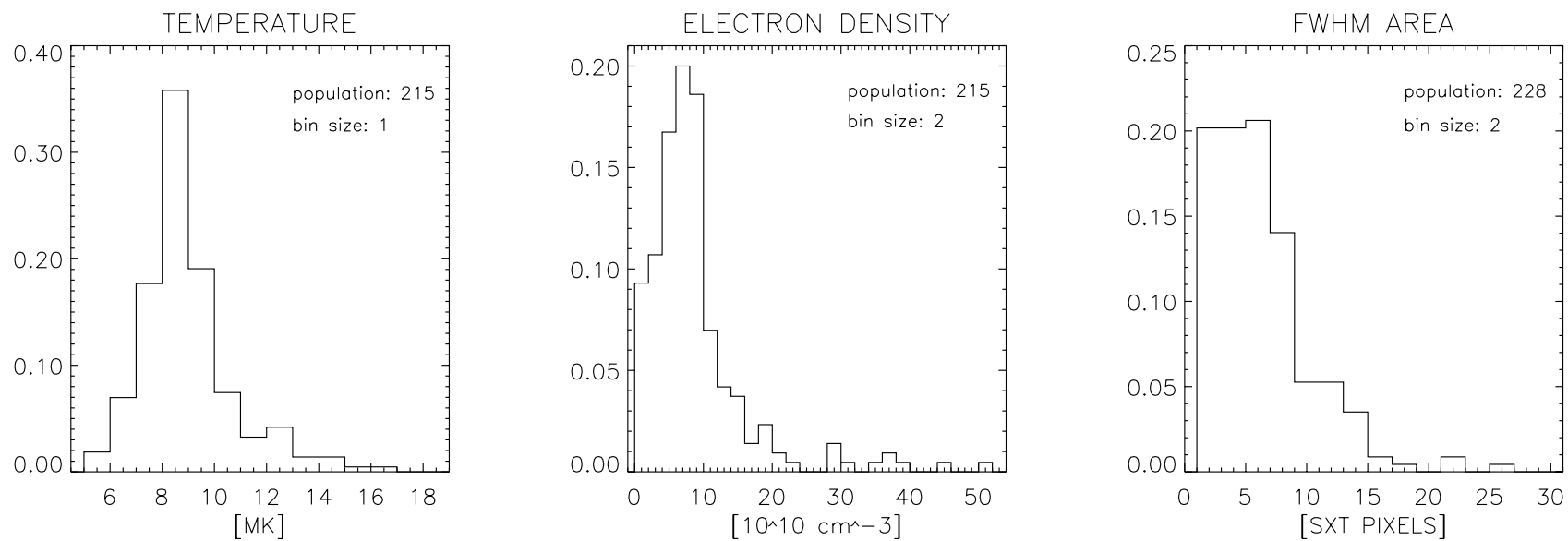

Fig. 8. Normalized histograms of the temperature, the electron density, and the FWHM area (left, middle, and right panel, respectively) for the investigated impulsive SXR brightenings.

The brightenings responsible for the maximum of about $100 \mathrm{~s}$ in the histogram of the FWHM duration do not form any separate class of events. We compared characteristics (2)-(9) for these events and for other brightenings but did not find any clear difference.
Longer durations are typical for flares with more complex impulsive phase consisting of many HXR bursts. We presume that such cases are caused by overlapping of the SXR responses to several consecutive non-thermal electron beams. The problem with the separation of individual impulsive SXR 
brightenings is caused not only by the poor time resolution of the SXT, but rather it is the result of the inertia of chromospheric response to non-thermal electron beams. If the interval between two consecutive beams is relatively short, then the enhancements of the temperature and electron density responsible for the impulsive SXR brightening overlap and we see only one event. Such a scenario is confirmed by the flares for which we only have observations in the Be119 filter but with better temporal resolution (Nos. 19-21, 28 and 40 in Table 1). We observed several HXR bursts for them, but even with 2-second time resolution we were unable to separate individual impulsive SXR brightenings.

In the histograms of the ratios $t_{\mathrm{r}} / t$ and $t_{\mathrm{d}} / \mathrm{t}$ (Fig. 6, middle and right panel, respectively) almost $90 \%$ of the population is concentrated within the interval of $0.3-0.7$, with a clear maximum near the value of 0.5 , suggesting that the SXR impulsive brightenings have symmetrical time profiles. Several values outside this interval are related to complex light curves for which we could not resolve the individual SXR responses.

Usually, at the beginning of the impulsive phase the footpoints are clearly visible. However, in the histogram of relative brightness (Fig. 7, left panel) in more than $85 \%$ of all cases it does not exceed the value of 0.1 . Such a disagreement is understandable if we remember that the impulsive SXR brightenings occur in several regions simultaneously and their summed brightness easily exceeds $20 \%-30 \%$ of the total SXR brightness of a flare.

The next histogram (Fig. 7, middle panel) presents the delay of the maximum of the SXR net light curve relative to the HXR light curve peak. Almost half of the population have values below $20 \mathrm{~s}$, which is similar to the SXT temporal resolution determined by the time interval between two exposures with the same filter. This means that the reaction of the chromosphere to the non-thermal electron beams is almost immediate. The second half of the events show a larger delay, in several cases even exceeding $100 \mathrm{~s}$. Neglecting some complex events, we can conclude that such large delays are the manifestation of the inertia of chromospheric response to non-thermal electrons.

We fitted a Poisson distribution to this histogram (the dotted line) and obtained the characteristic time scale of separation between the HXR and SXR response to be $=35 \pm 3 \mathrm{~s}$. This result agrees very well with the chromospheric evaporation time scale obtained from numerical simulations of flare loops (e.g. Antonucci et al. 1993; Hori et al. 1998).

In the histogram containing ratios $t / t_{\mathrm{HXR}}$ (Fig. 7 , right panel) of the FWHM duration of the impulsive SXR brightening, $t$, and of the HXR burst, $t_{\mathrm{HXR}}$, the majority of events ( $\sim 90 \%)$ falls between values 1 and 9 with the maximum between 2 and 3. The longer duration of the impulsive SXR brightenings than that of the HXR burst is another example of the inertia of chromospheric response to non-thermal electrons.

The investigated footpoints have relatively low temperatures, usually they are the coolest part of flaring structures. The histogram in Fig. 8 (left panel) has a clear maximum within the interval 8-9 MK. A small group of events in the histogram with temperatures above $10 \mathrm{MK}$ comprises the geometrical configurations in which the footpoint and the hotter, bright loop-top kernel partly overlap.
More than half the investigated footpoints show electron densities in the interval of $4-10 \times 10^{10} \mathrm{~cm}^{-3}$. A similarity between values obtained for disk events and for limb flares proves that the effect of overlap of the chromospheric with the coronal part is negligible. During the impulsive phase the footpoints are the most dense fragment of flaring structures.

The last value presented in the form of a histogram is the FWHM area of the regions of SXR response (Fig. 8, right panel). For about $75 \%$ of events the values are within the interval of 1-9 SXT pixels. This may suggest that the actual areas of footpoints were below the spatial resolution of the SXT. A small group of large footpoints with sizes above 15 pixels was probably produced by several smaller events spread by the PSF of the SXT.

For some regions of the SXR response a systematic trend has been found. Namely, the moment of the impulsive SXR brightening maximum depends on the situation in the flaring structure - light curves of the pixels located higher above the limb show their maximum later in time. Regarding the relatively low temperatures and relatively high densities of the footpoints, we conclude that impulsive SXR brightenings are caused by the inflow of dense plasma into the flaring structure i.e. by chromospheric evaporation. We estimated velocities of the evaporation comparing the time of the maximum brightness with the position along the structure (see Tomczak 1997). The obtained values, presented in Table 1, are within the interval of $130-720 \mathrm{~km} \mathrm{~s}^{-1}$. The method makes it possible to measure only the transversal component of the velocity vector. Therefore it works best for the limb flares.

\subsection{Comparison of two kinds of $X$-ray response in flare footpoints}

We compared the SXR response to non-thermal electrons in flare footpoints to the HXR emission characteristics. This kind of analysis was possible only for a small fraction of 228 events. First, for some flares (11 events) there were no HXT observations due to insufficient sensivity of the telescope or due to the South Atlantic Anomaly passage of the satellite. Second, the low dynamical range of the HXT made the HXR photometry meaningless for faint sources in the presence of strong ones (e.g. footpoint F4 in Fig. 1). Third, we needed values of the HXR flux in at least two HXT channels to estimate the photon energy spectrum.

Taking this into account we were able to make the comparison between the SXR and HXR response to non-thermal electrons for 37 events from 18 flares (see Table 2). As we see, the HXR photometry is based on channels $M 1$ and $M 2$. In channel $L$ we often could not exclude thermal emission of the flaring structure. We included fluxes in this channel only for the sources which showed the same size in channel $L$ as in the higher-energy channels. For many flares the HXT did not detect any signal above the background in channel $H$.

We estimated the HXR response at the maximum of strong bursts where we expect the strongest flux of non-thermal electrons. The SXR response refers to the maximum of the SXR net light curve which corresponds to the HXR burst. 
Table 2. Response to the non-thermal electron beams in the investigated footpoints recorded by the SXT and the HXT.

\begin{tabular}{|c|c|c|c|c|c|c|c|c|c|c|c|c|c|}
\hline \multirow{3}{*}{ No. ${ }^{a}$} & \multirow{3}{*}{ Date } & \multicolumn{3}{|c|}{ SXT(Al12) } & \multicolumn{9}{|c|}{ HXT } \\
\hline & & \multirow{2}{*}{$\begin{array}{l}\text { Time } \\
\text { [UT] }\end{array}$} & \multirow{2}{*}{$\begin{array}{c}\text { Signal } \\
{\left[\mathrm{DN} \mathrm{s}^{-1}\right]}\end{array}$} & \multirow{2}{*}{$\begin{array}{c}E_{\mathrm{th}} \\
{[\mathrm{ergs}]}\end{array}$} & \multirow{2}{*}{$\begin{array}{l}\text { Time } \\
\text { [UT] }\end{array}$} & \multirow[t]{2}{*}{$L$} & \multirow{2}{*}{$\frac{M 1}{\text { cts s }^{-1}}$} & $M 2$ & $H$ & $\gamma$ & $A$ & & $E_{0}$ \\
\hline & & & & & & & & $1 \mathrm{~cm}^{-2}$ & & & & [ergs] & {$[\mathrm{keV}]$} \\
\hline 1. & $24-O C T .-91$ & $22: 36: 36$ & $8.0 \times 10^{5}$ & $5.6 \times 10^{29}$ & $22: 36: 21.1$ & $\ldots$ & 187.4 & 68.2 & 13.8 & 4.7 & $1.5 \times 10^{8}$ & $1.9 \times 10^{30}$ & 20 \\
\hline 1.1 & & $22: 36: 42$ & $4.6 \times 10^{5}$ & $2.0 \times 10^{29}$ & $22: 36: 21.1$ & $\ldots$ & 64.6 & 23.0 & 4.8 & 4.7 & $5.5 \times 10^{7}$ & $9.8 \times 10^{29}$ & 20 \\
\hline $2 .^{\mathrm{a}}$ & $02-N O V .-91$ & $06: 45: 48$ & $6.7 \times 10^{5}$ & $6.4 \times 10^{29}$ & $06: 45: 45.1$ & $\ldots$ & 426.4 & 276.3 & 126.0 & 3.2 & $2.5 \times 10^{6}$ & $2.1 \times 10^{30}$ & 24 \\
\hline $2 .{ }^{a} 1$ & & $06: 45: 46$ & $3.8 \times 10^{5}$ & $4.4 \times 10^{29}$ & $06: 45: 45.1$ & $\ldots$ & 155.9 & 102.5 & 41.0 & 3.3 & $1.3 \times 10^{6}$ & $1.1 \times 10^{30}$ & 20 \\
\hline $2 .^{\mathrm{b}}$ & & $06: 47: 02$ & $7.2 \times 10^{5}$ & $6.3 \times 10^{29}$ & $06: 46: 22.6$ & $\ldots$ & 249.8 & 108.6 & 27.0 & 4.2 & $5.6 \times 10^{6}$ & $2.5 \times 10^{29}$ & 11 \\
\hline $2 .^{\mathrm{b}} 1$ & & $06: 46: 57$ & $2.8 \times 10^{5}$ & $3.1 \times 10^{29}$ & $06: 46: 22.6$ & $\ldots$ & 21.3 & 9.1 & 2.9 & 4.1 & $3.1 \times 10^{6}$ & $2.3 \times 10^{29}$ & 12 \\
\hline $2 .{ }^{b} 2$ & & 06:47:02 & $4.4 \times 10^{5}$ & $2.8 \times 10^{29}$ & $06: 46: 22.6$ & . & 50.1 & 23.1 & 5.9 & 3.9 & $3.8 \times 10^{6}$ & $4.6 \times 10^{29}$ & 16 \\
\hline $4 .^{\mathrm{a}}$ & $10-N O V .-91$ & $20: 07: 58$ & $1.5 \times 10^{6}$ & $6.4 \times 10^{29}$ & $20: 07: 17.1$ & 176.3 & 153.5 & 103.7 & 57.1 & 3.3 & $4.2 \times 10^{5}$ & $4.7 \times 10^{29}$ & 12 \\
\hline $4 .{ }^{\mathrm{a}} 1$ & & 20:08:08 & $4.4 \times 10^{5}$ & $4.8 \times 10^{29}$ & $20: 07: 17.4$ & 47.2 & 57.7 & 38.2 & 16.2 & 3.2 & $1.1 \times 10^{5}$ & $3.3 \times 10^{29}$ & 20 \\
\hline $4 . \mathrm{b}$ & & 20:09:30 & $9.7 \times 10^{5}$ & $4.3 \times 10^{29}$ & $20: 09: 24.5$ & $\ldots$ & 203.0 & 155.9 & 115.7 & 2.6 & $1.2 \times 10^{5}$ & $2.2 \times 10^{29}$ & 9 \\
\hline $4 .{ }^{\mathrm{b}} 1$ & & $20: 09: 25$ & $5.7 \times 10^{5}$ & $1.7 \times 10^{29}$ & $20: 09: 24.5$ & 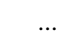 & 134.3 & 110.0 & 69.1 & 2.6 & $1.5 \times 10^{5}$ & $1.5 \times 10^{29}$ & 8 \\
\hline 5. & $19-N O V .-91$ & 09:29:28 & $3.5 \times 10^{5}$ & $3.8 \times 10^{29}$ & $09: 28: 37.0$ & 21.4 & 13.8 & 4.7 & $\ldots$ & 4.7 & $1.4 \times 10^{7}$ & $2.7 \times 10^{30}$ & 24 \\
\hline 5.1 & & 09:29:39 & $7.0 \times 10^{4}$ & $2.0 \times 10^{28}$ & $09: 28: 37.0$ & 5.1 & 3.7 & 1.1 & $\ldots$ & 5.0 & $1.0 \times 10^{7}$ & $1.2 \times 10^{29}$ & 20 \\
\hline 5.2 & & 09:29:09 & $4.0 \times 10^{4}$ & $2.5 \times 10^{28}$ & $09: 28: 37.0$ & 1.2 & 1.2 & 0.3 & $\ldots$ & 5.8 & $3.4 \times 10^{7}$ & $7.8 \times 10^{28}$ & 17 \\
\hline 6. & $13-J A N .-92$ & $17: 28: 37$ & $3.6 \times 10^{5}$ & $2.6 \times 10^{29}$ & $17: 28: 20.3$ & $\ldots$ & 27.5 & 15.3 & 6.6 & 3.4 & $3.7 \times 10^{5}$ & $3.8 \times 10^{29}$ & 17 \\
\hline 6.1 & & $17: 28: 37$ & $2.5 \times 10^{5}$ & $7.2 \times 10^{28}$ & $17: 28: 20.3$ & $\ldots$ & 7.7 & 5.2 & 1.7 & 3.5 & $1.6 \times 10^{5}$ & $1.4 \times 10^{29}$ & 17 \\
\hline 6.2 & & $17: 28: 25$ & $1.2 \times 10^{5}$ & $1.2 \times 10^{29}$ & $17: 28: 20.3$ & $\ldots$ & 11.4 & 6.5 & 2.7 & 3.4 & $1.6 \times 10^{5}$ & $1.9 \times 10^{29}$ & 24 \\
\hline 7. & $26-J A N .-92$ & $15: 28: 40$ & $2.1 \times 10^{6}$ & $1.7 \times 10^{30}$ & $15: 28: 34.6$ & $\ldots$ & 696.2 & 398.3 & 113.0 & 3.7 & $2.9 \times 10^{7}$ & $4.3 \times 10^{30}$ & 20 \\
\hline 7.1 & & $15: 28: 37$ & $6.2 \times 10^{5}$ & $5.0 \times 10^{28}$ & $15: 28: 34.6$ & $\ldots$ & 197.3 & 107.7 & 30.7 & 3.5 & $3.7 \times 10^{6}$ & $1.1 \times 10^{30}$ & 44 \\
\hline 7.2 & & $15: 28: 41$ & $1.0 \times 10^{6}$ & $6.1 \times 10^{29}$ & $15: 28: 34.6$ & $\ldots$ & 214.0 & 120.4 & 33.4 & 3.4 & $3.1 \times 10^{6}$ & $1.1 \times 10^{30}$ & 16 \\
\hline 7.3 & & $15: 29: 07$ & $3.1 \times 10^{5}$ & $2.9 \times 10^{28}$ & $15: 28: 34.6$ & $\ldots$ & 35.4 & 21.4 & 6.9 & 3.2 & $3.0 \times 10^{5}$ & $1.5 \times 10^{29}$ & 27 \\
\hline 8. & $14-F E B .-92$ & $23: 07: 50$ & $4.4 \times 10^{6}$ & $2.1 \times 10^{30}$ & 23:07:30.1 & $\ldots$ & 974.8 & 554.3 & 195.5 & 3.6 & $2.2 \times 10^{7}$ & $3.9 \times 10^{30}$ & 18 \\
\hline 8.1 & & 23:07:58 & $1.1 \times 10^{6}$ & $2.7 \times 10^{29}$ & 23:07:30.1 & $\ldots$ & 327.9 & 180.9 & 59.0 & 3.7 & $1.1 \times 10^{7}$ & $2.0 \times 10^{30}$ & 27 \\
\hline 8.2 & & $23: 07: 40$ & $6.3 \times 10^{5}$ & $7.3 \times 10^{28}$ & $23: 07: 30.1$ & . & 282.3 & 163.7 & 52.9 & 3.6 & $7.6 \times 10^{6}$ & $1.6 \times 10^{30}$ & 43 \\
\hline 8.3 & & $23: 07: 46$ & $1.6 \times 10^{6}$ & $2.0 \times 10^{29}$ & 23:07:30.1 & & 91.1 & 41.0 & 13.7 & 3.9 & $7.1 \times 10^{6}$ & $6.6 \times 10^{29}$ & 20 \\
\hline $9 .^{\mathrm{a}}$ & $17-F E B .-92$ & $15: 40: 55$ & $2.8 \times 10^{5}$ & $1.4 \times 10^{29}$ & $15: 40: 53.1$ & 25.9 & 15.0 & 6.6 & $\ldots$ & 4.1 & $1.7 \times 10^{6}$ & $1.1 \times 10^{29}$ & 13 \\
\hline $9 .{ }^{\mathrm{a}} 1$ & & $15: 40: 53$ & $1.4 \times 10^{5}$ & $1.8 \times 10^{29}$ & $15: 40: 53.1$ & 6.1 & 3.5 & 1.6 & $\ldots$ & 4.0 & $3.0 \times 10^{5}$ & $3.3 \times 10^{28}$ & 12 \\
\hline $9 .{ }^{a} 2$ & & 15:41:07 & $7.0 \times 10^{4}$ & $3.0 \times 10^{28}$ & $15: 40: 53.1$ & 10.1 & 6.8 & 2.8 & $\ldots$ & 4.3 & $1.5 \times 10^{6}$ & $8.0 \times 10^{28}$ & 24 \\
\hline $9 .^{\mathrm{b}}$ & & $15: 42: 35$ & $2.6 \times 10^{5}$ & $2.3 \times 10^{29}$ & $15: 42: 06.1$ & 29.8 & 17.3 & 10.2 & $\ldots$ & 3.3 & $1.7 \times 10^{5}$ & $3.1 \times 10^{28}$ & 6 \\
\hline $9 .{ }^{\mathrm{b}} 1$ & & $15: 42: 39$ & $1.8 \times 10^{5}$ & $1.7 \times 10^{29}$ & $15: 42: 06.1$ & 2.7 & 1.6 & 1.1 & $\ldots$ & 3.0 & $1.1 \times 10^{4}$ & $2.3 \times 10^{28}$ & 3 \\
\hline $9 .{ }^{b} 2$ & & $15: 42: 25$ & $6.0 \times 10^{4}$ & $3.0 \times 10^{28}$ & $15: 42: 06.1$ & 17.6 & 9.7 & 5.4 & $\ldots$ & 3.5 & $1.6 \times 10^{5}$ & $2.3 \times 10^{28}$ & 17 \\
\hline $11 .^{\mathrm{a}}$ & $08-J U L .-92$ & 09:46:00 & $5.1 \times 10^{5}$ & $3.6 \times 10^{29}$ & $09: 45: 56.3$ & $\ldots$ & 334.2 & 164.2 & 50.3 & 3.9 & $2.2 \times 10^{7}$ & $1.4 \times 10^{30}$ & 22 \\
\hline $11 .^{\mathrm{a}} 1$ & & 09:46:02 & $1.3 \times 10^{5}$ & $1.1 \times 10^{29}$ & 09:45:56.3 & $\ldots$ & 136.1 & 68.7 & 19.4 & 3.9 & $9.8 \times 10^{6}$ & $7.3 \times 10^{29}$ & 25 \\
\hline 11. ${ }^{\mathrm{2}} 2$ & & 09:45:58 & $2.9 \times 10^{5}$ & $8.0 \times 10^{28}$ & $09: 45: 56.3$ & $\ldots$ & 58.6 & 29.2 & 9.6 & 3.8 & $3.0 \times 10^{6}$ & $4.7 \times 10^{29}$ & 25 \\
\hline $11^{\mathrm{b}}$ & & 09:46:32 & $1.1 \times 10^{6}$ & $5.3 \times 10^{29}$ & $09: 46: 32.3$ & $\ldots$ & 492.4 & 244.7 & 91.0 & 3.7 & $1.8 \times 10^{7}$ & $3.0 \times 10^{30}$ & 27 \\
\hline $11^{\mathrm{b}} 1$ & & 09:46:32 & $3.6 \times 10^{5}$ & $2.0 \times 10^{29}$ & $09: 46: 32.3$ & $\ldots$ & 152.0 & 76.3 & 25.2 & 3.8 & $7.3 \times 10^{6}$ & $8.8 \times 10^{29}$ & 22 \\
\hline 12. & $15-J U L .-92$ & 02:00:12 & $1.5 \times 10^{5}$ & $2.4 \times 10^{29}$ & 02:00:04.1 & 17.4 & 8.6 & 3.9 & $\ldots$ & 4.8 & $9.7 \times 10^{6}$ & $6.1 \times 10^{28}$ & 10 \\
\hline 12.1 & & 02:00:04 & $2.3 \times 10^{4}$ & $8.4 \times 10^{28}$ & 02:00:04.1 & 6.4 & 2.7 & 1.0 & $\ldots$ & 5.2 & $1.3 \times 10^{7}$ & $3.9 \times 10^{28}$ & 11 \\
\hline 12.2 & & 02:00:12 & $3.6 \times 10^{4}$ & $1.4 \times 10^{28}$ & 02:00:04.1 & 4.7 & 2.5 & 1.3 & $\ldots$ & 4.5 & $1.3 \times 10^{6}$ & $2.1 \times 10^{28}$ & 15 \\
\hline 13. & $11-A U G .-92$ & $13: 48: 30$ & $4.1 \times 10^{5}$ & $2.1 \times 10^{29}$ & $13: 47: 44.3$ & 64.2 & 59.4 & 31.2 & $\ldots$ & 3.6 & $1.5 \times 10^{6}$ & $1.8 \times 10^{29}$ & 13 \\
\hline 13.1 & & $13: 47: 45$ & $2.1 \times 10^{4}$ & $4.1 \times 10^{28}$ & $13: 47: 44.3$ & 21.4 & 21.6 & 10.9 & $\ldots$ & 3.7 & $6.4 \times 10^{5}$ & $9.1 \times 10^{28}$ & 16 \\
\hline 13.2 & & $13: 48: 37$ & $3.5 \times 10^{5}$ & $7.5 \times 10^{28}$ & $13: 47: 44.3$ & 20.9 & 20.2 & 9.7 & $\ldots$ & 3.8 & $1.1 \times 10^{6}$ & $1.0 \times 10^{29}$ & 14 \\
\hline 16. & $06-S E P .-92$ & 09:04:25 & $7.5 \times 10^{5}$ & $5.8 \times 10^{29}$ & 09:03:32.5 & 99.0 & 55.4 & 21.1 & $\ldots$ & 4.4 & $2.1 \times 10^{7}$ & $3.1 \times 10^{29}$ & 12 \\
\hline 16.1 & & 09:04:25 & $6.3 \times 10^{5}$ & $2.6 \times 10^{29}$ & $09: 03: 32.5$ & 38.2 & 20.3 & 7.3 & $\ldots$ & 4.6 & $1.3 \times 10^{7}$ & $1.6 \times 10^{29}$ & 11 \\
\hline 16.2 & & 09:04:20 & $7.0 \times 10^{4}$ & $2.0 \times 10^{28}$ & 09:03:32.5 & 27.0 & 19.6 & 6.2 & $\ldots$ & 4.9 & $3.9 \times 10^{7}$ & $2.2 \times 10^{29}$ & 25 \\
\hline 17. & $11-S E P .-92$ & 03:00:15 & $1.9 \times 10^{5}$ & $1.4 \times 10^{29}$ & $02: 59: 48.0$ & 38.0 & 22.5 & 10.4 & $\ldots$ & 4.2 & $4.7 \times 10^{6}$ & $1.2 \times 10^{29}$ & 13 \\
\hline 17.1 & & 03:00:10 & $1.2 \times 10^{5}$ & $5.1 \times 10^{28}$ & $02: 59: 48.0$ & 12.9 & 9.8 & 4.5 & $\ldots$ & 4.0 & $8.5 \times 10^{5}$ & $4.8 \times 10^{28}$ & 12 \\
\hline 18. & $12-S E P .-92$ & $15: 39: 20$ & $1.1 \times 10^{5}$ & $2.1 \times 10^{29}$ & $15: 38: 48.1$ & & 16.2 & 4.6 & $\ldots$ & 5.2 & $7.5 \times 10^{7}$ & $2.2 \times 10^{29}$ & 14 \\
\hline 18.1 & & 15:39:05 & $7.0 \times 10^{4}$ & $9.0 \times 10^{28}$ & $15: 38: 48.1$ & 5.4 & 3.4 & 1.3 & $\ldots$ & 5.8 & $1.2 \times 10^{8}$ & $1.1 \times 10^{29}$ & 14 \\
\hline 25. & $16-J A N .-94$ & $23: 17: 10$ & $2.9 \times 10^{5}$ & $5.1 \times 10^{29}$ & $23: 16: 48.6$ & $\ldots$ & 51.4 & 18.5 & 7.2 & 4.2 & $9.0 \times 10^{6}$ & $4.3 \times 10^{29}$ & 13 \\
\hline 25.1 & & $23: 17: 59$ & $6.0 \times 10^{4}$ & $1.4 \times 10^{29}$ & $23: 16: 48.6$ & $\ldots$ & 15.9 & 6.3 & 2.2 & 4.1 & $2.2 \times 10^{6}$ & $1.6 \times 10^{29}$ & 20 \\
\hline 25.2 & & $23: 16: 57$ & $6.0 \times 10^{4}$ & $4.0 \times 10^{28}$ & $23: 16: 48.6$ & & 8.1 & 3.3 & 1.1 & 4.1 & $1.1 \times 10^{6}$ & $9.4 \times 10^{28}$ & 19 \\
\hline 30. & $02-S E P .-97$ & $21: 11: 10$ & $5.0 \times 10^{5}$ & $5.0 \times 10^{28}$ & $21: 10: 49.2$ & 9.7 & 6.1 & 2.1 & $\ldots$ & 4.7 & $5.5 \times 10^{6}$ & $8.3 \times 10^{28}$ & 16 \\
\hline 30.1 & & $21: 11: 15$ & $3.0 \times 10^{4}$ & $1.0 \times 10^{28}$ & $21: 10: 49.2$ & 4.0 & 2.8 & 0.8 & $\ldots$ & 5.2 & $1.2 \times 10^{7}$ & $8.2 \times 10^{28}$ & 21 \\
\hline 31. & $14-S E P .-97$ & $02: 54: 25$ & $1.7 \times 10^{5}$ & $2.1 \times 10^{29}$ & 02:53:29.9 & 21.6 & 14.7 & 5.9 & $\ldots$ & 4.3 & $3.6 \times 10^{6}$ & $1.7 \times 10^{29}$ & 13 \\
\hline 31.1 & & $02: 55: 03$ & $5.0 \times 10^{4}$ & $2.0 \times 10^{28}$ & $02: 53: 29.9$ & 9.8 & 7.4 & 2.6 & & 4.7 & $6.2 \times 10^{6}$ & $1.6 \times 10^{29}$ & 22 \\
\hline 31.2 & & $02: 54: 13$ & $8.0 \times 10^{4}$ & $6.0 \times 10^{28}$ & $02: 53: 29.9$ & 3.8 & 2.5 & 1.1 & $\ldots$ & 4.1 & $3.5 \times 10^{5}$ & $3.3 \times 10^{28}$ & 12 \\
\hline $44 .^{\mathrm{a}}$ & $28-M A Y-98$ & 19:04:02 & $1.0 \times 10^{5}$ & $1.8 \times 10^{29}$ & 19:03:06.4 & 10.0 & 9.1 & 5.4 & 2.0 & 3.5 & $1.5 \times 10^{5}$ & $2.6 \times 10^{28}$ & 6 \\
\hline $44 .{ }^{\mathrm{a}} 1$ & & 19:04:25 & $2.0 \times 10^{4}$ & $1.0 \times 10^{28}$ & 19:03:06.4 & 4.6 & 4.2 & 2.5 & 0.9 & 3.5 & $8.7 \times 10^{5}$ & $1.7 \times 10^{28}$ & 18 \\
\hline 44..$^{\mathrm{b}}$ & & 19:06:58 & $4.4 \times 10^{5}$ & $2.0 \times 10^{29}$ & $19: 06: 22.0$ & $\ldots$ & 4.1 & 1.5 & & 4.5 & $2.2 \times 10^{6}$ & $2.3 \times 10^{28}$ & 8 \\
\hline $44 .^{b} 1$ & & $19: 06: 29$ & $1.2 \times 10^{5}$ & $4.4 \times 10^{28}$ & 19:06:22.0 & $\ldots$ & 1.8 & 0.5 & $\ldots$ & 5.3 & $1.0 \times 10^{7}$ & $1.4 \times 10^{28}$ & 11 \\
\hline
\end{tabular}

a The numbers of flares are the same as in Table 1. Digits after the number of the event refer to different footpoints, letters refer to different HXR bursts. 

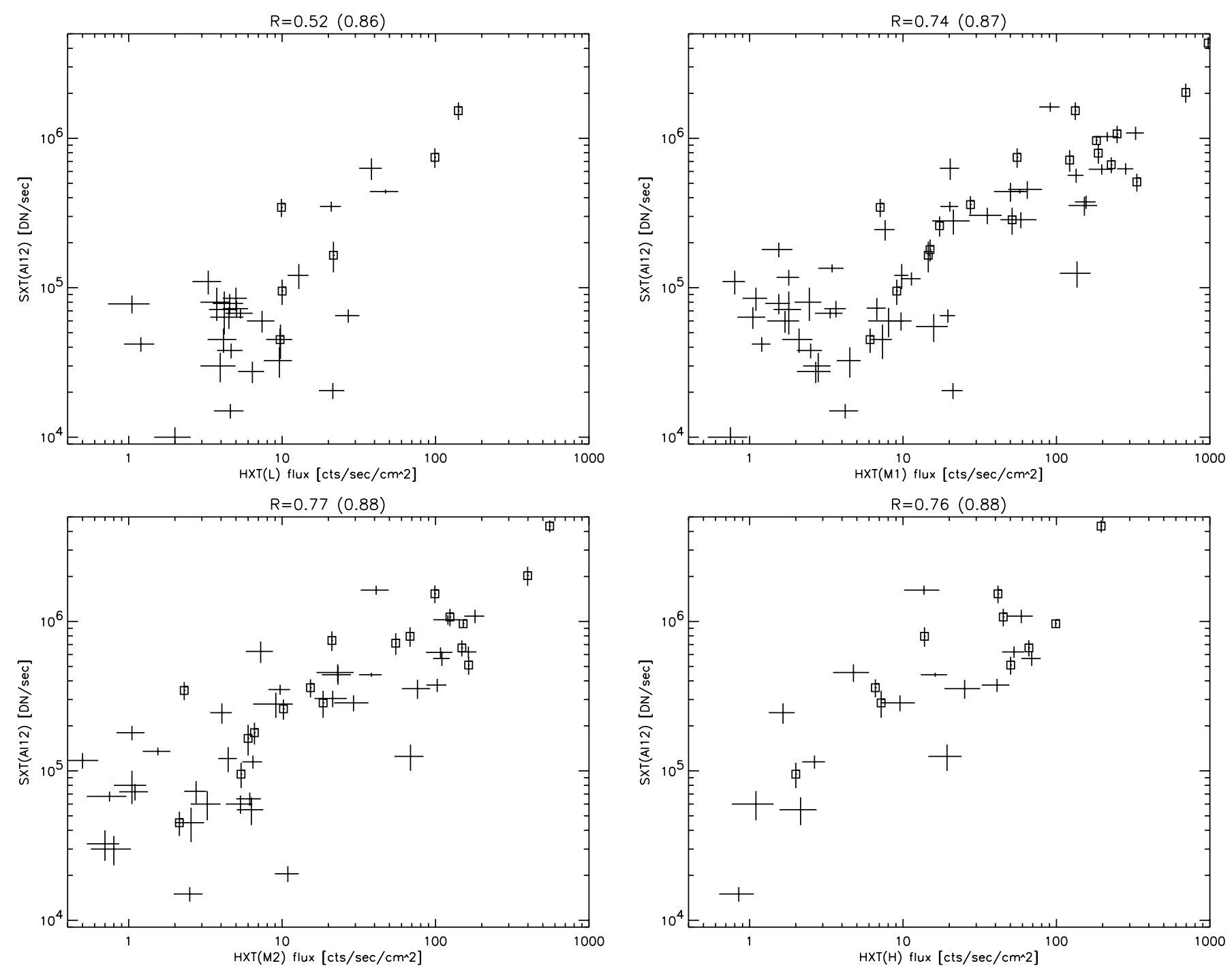

Fig. 9. Relation between two kinds of response to the non-thermal electron beams in footpoints observed in $\operatorname{HXT}(L, M 1, M 2$ and $H$, respectively) channels and SXT(A112) filter. R is the correlation coefficient. Total values for the complete events have been marked by squares. Values of the correlation coefficient for them are given in parentheses.

The response values presented in Table 2 are the averaged values of maximal and minimal values that were estimated as follows. For SXRs, the lower limit is the value of the maximum of the net light curve i.e. the light curve with the gradual component subtracted. The upper limit is the value of the maximum of the total light curve obtained for the impulsive SXR brightening investigated. For HXR emission sources, the lower limit is simply the total flux from all pixels within the determined borders of a HXR source. The upper limit was calculated considering all faint sources (having an intensity below $10 \%$ of the most intense pixel) as spurious i.e. produced by the reconstruction method (Metcalf et al. 1996; Alexander \& Metcalf 1997). We redistributed their signal proportionally between the strong sources (Tomczak 1999). The obtained ranges of uncertainties can be considered as maximal errors of the estimations.

To verify the correctness of the reconstruction of the HXT images we defined complete responses for events in which all stronger HXR sources (above 10\%-limit) were of the footpointtype. In such cases we took the total HXR flux minus the background as the HXR response and the total signal from net SXR light curves for all footpoints as the SXR response. The complete responses are also included in Table 2.

Figure 9 shows the relation between the HXR response in the HXT channels and the SXR response recorded in the Al12 filter. The values are taken from Table 2 with $1 \sigma$ error bars. We can see evident correlation of the SXRs with HXT channels $M 1, M 2$, and $H$, described with the correlation coefficients, $R$, equal to $0.74,0.77$, and 0.76 , respectively. Such a strong correlation proves the common physical origin of SXR and HXR responses, namely the non-thermal electron beams. In channel $L$ the correlation is less good $(R=0.52)$. We presume that this is caused by a contribution of thermal emission in HXRs. The points representing values for complete events, marked by squares in Fig. 9, show even better correlation $(0.86,0.87,0.88$ and 0.88 respectively) than those calculated for all investigated footpoints. The main reason of this difference is probably an additional source of errors introduced by the HXR image reconstruction method. 

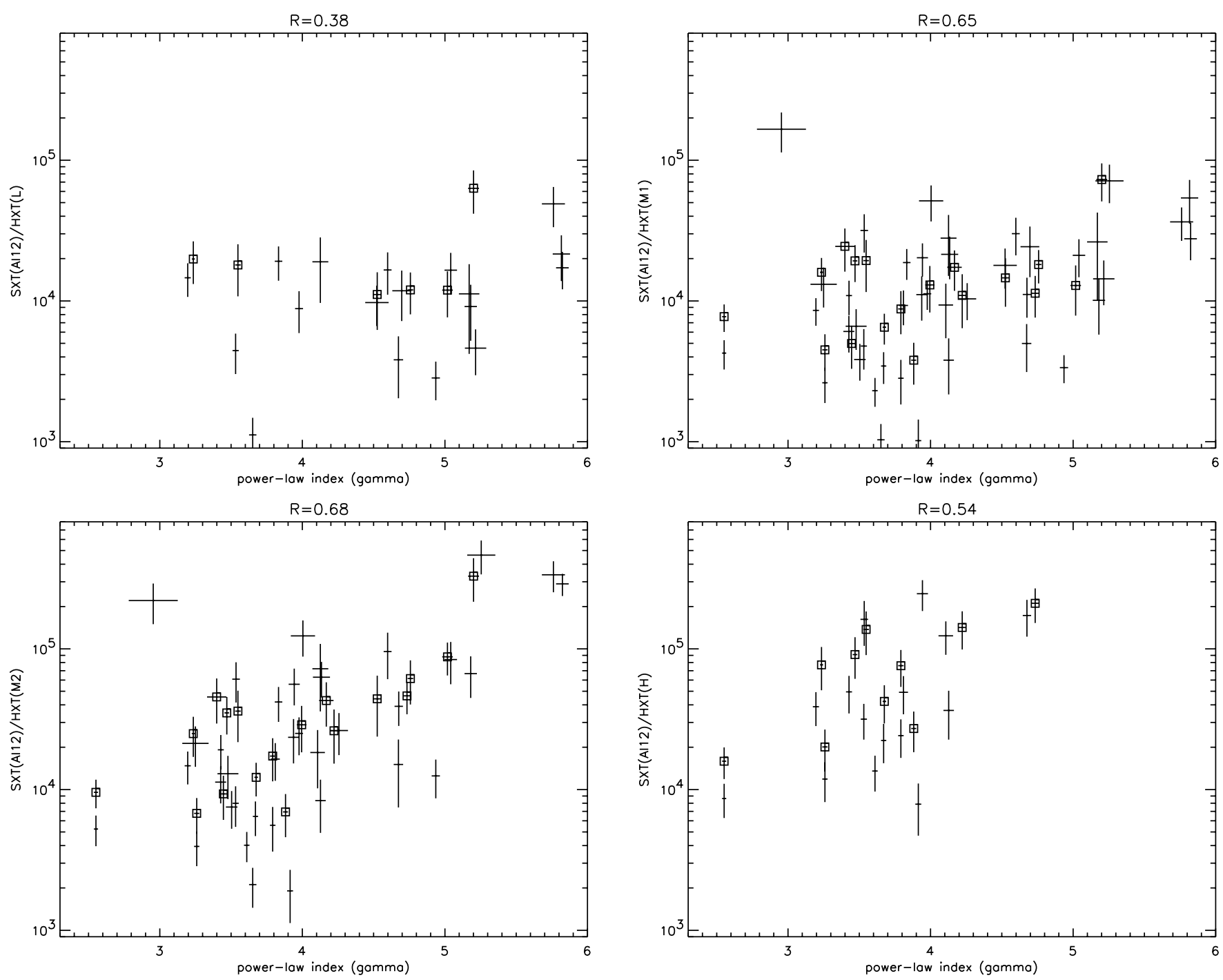

Fig. 10. Plot of productivity of soft X-rays SXT(Al12) relative to hard X-rays (HXT channels: $L, M 1, M 2$ and $H$, respectively) and power-law indices that were taken from the single power-law fit. $R$ is the correlation coefficient. Total values for the complete events have been marked by squares.

There is a scatter, seen in Fig. 9, which cannot be caused only by observational uncertainties and systematical errors. Points representing individual footpoints of the same event are often situated along a line that is almost perpendicular to the general trend. This effect could be seen in Fig. 1 where the footpoint $\mathrm{F} 2$ seen brighter in SXRs shows the lower HXR emission, while the footpoint F3 seen fainter in SXRs shows the stronger HXR emission.

Tomczak (1999) showed that the scatter in the relation between the HXR response and the SXR response is caused by the photon energy spectrum. Assuming the thick-target model, it means that the relation between the HXR and SXR response depends on the energy spectrum of non-thermal electron beams.

We used the values from Table 2 to make a single powerlaw fit using the following formula:

$I(E)=A E^{-\gamma}$ photons $\mathrm{cm}^{-2} \mathrm{~s}^{-1} \mathrm{keV}^{-1}$ where $I$ is the photon flux at energy $E, \gamma$ is the power-law index and $A$ is a constant. Keeping in mind a thermal contribution to channel $L$, we excluded this channel from the determination of photon energy spectra. The results of the fit $(\gamma$ and $A)$ are presented in Table 2.

In Fig. 10 we present the relation between the relative productivity of the SXRs with regard to HXRs (the ratio of the SXR and HXR response) and the power-law index, $\gamma$. As we see, the correlation is quite good for channels $M 1, M 2$, and $H$ (the correlation coefficient is equal to $0.65,0.68$, and 0.54 , respectively). As in Fig. 9, the correlation for channel $L$ is distinctly worse and this is caused by the thermal contribution to this channel.

An increase of the coefficient $\gamma$ in Eq. (1) makes the powerlaw steeper which implies an enhancement of the number of low-energy photons and electrons producing them with regard to high-energy ones. Therefore, the increase of the relative productivity of SXRs with regard to HXRs, for higher values of the 


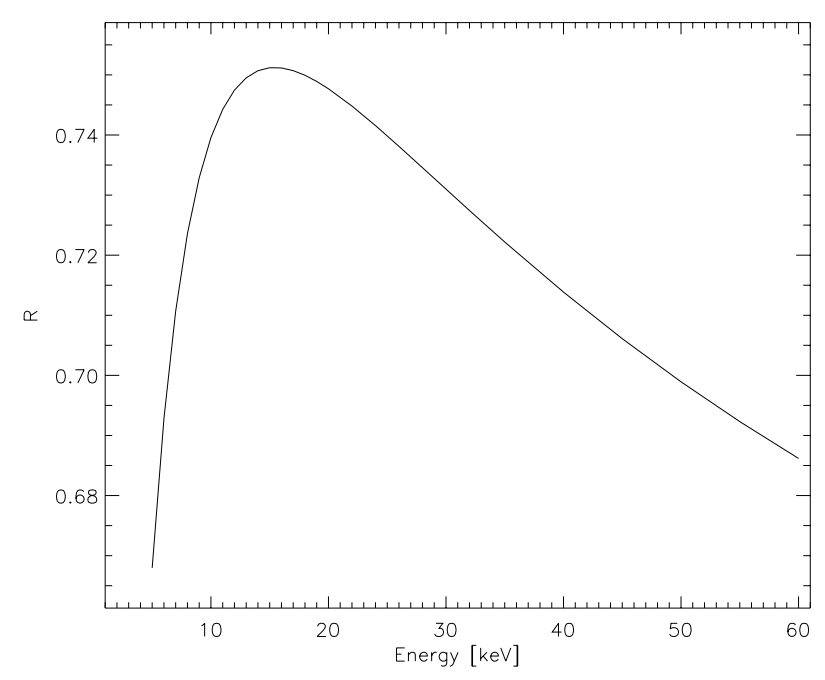

Fig. 11. The correlation coefficient, $R$, between the HXR flux [photons s $\mathrm{s}^{-1} \mathrm{~cm}^{-2} \mathrm{keV}^{-1}$ ] and the SXR response [DN s${ }^{-1}$ ] as a function of the photon energy $[\mathrm{keV}]$.

power-law index, means that non-thermal electrons with relatively low energies should play a dominant role in the generation of impulsive SXR brightenings.

However, only the two parameters, $\gamma$ and $A$, make it possible to estimate the actual number of HXR photons at particular energies. Using the values presented in Table 2 we calculated the number of HXR photons for each footpoint and for several values of the energy. For each value of the energy we plotted the number of HXR photons versus the SXR response from Table 2 and calculated the correlation coefficient, $R$. The dependence of $R$ on different energies is presented in Fig. 11. As we see, the best correlation is for the range of $13-17 \mathrm{keV}$. We interpret this result as an additional proof that impulsive SXR brightenings are generated mostly by non-thermal electrons having relatively low energies. Using the Bethe-Heitler formula we estimated the energy interval of non-thermal electrons to be approximately between 13 and $50 \mathrm{keV}$.

The process of chromospheric evaporation driven by nonthermal electron beams is controlled by the energy balance. The energy deposited by the non-thermal electrons, $E_{\mathrm{nth}}$, should be equal to the maximum thermal energy, $E_{\mathrm{th}}^{\max }$, contained in the plasma that is heated by this electron population,

$E_{\text {nth }}=E_{\text {th }}^{\max }$.

The X-ray emissions are easier to record than the energies. The HXR emission is a measure of the rate of energy deposition by non-thermal electrons and the SXR emission is a measure of the total energy contained in the plasma heated by thermalization of these electrons. However, the HXR and SXR emissions are not directly indicative of the energies involved - energies are not simply linearly related to emissions. Therefore, the Neupert effect should exist between the energies rather than between the X-ray emissions (Lee et al. 1995).

We calculated the energies $E_{\mathrm{nth}}$ and $E_{\mathrm{th}}^{\mathrm{max}}$ for the footpoints investigated as follows. We used the formula,

$E_{\text {nth }}=0.5 F\left(\geq E_{0}\right) \Delta t$ ergs, for the total, time-integrated energy deposited by non-thermal electrons, where $\Delta t$ is the duration of impulsive increase and $F\left(E \geq E_{0}\right)$ is the total energy flux of non-thermal electrons above the cut-off energy $E_{0}$. We choose the energy $E_{0}=$ $14 \mathrm{keV}$. According to the thick-target model (Crosby et al. 1993):

$F\left(\geq E_{0}\right)=k A E^{-\gamma+1} \gamma(\gamma-1) B(\gamma-0.5,0.5) \mathrm{ergs} \mathrm{s}^{-1}$

where $B$ is the beta function and $k$ is a constant equal to $4.8 \times$ $10^{24}$.

We estimated the maximum thermal energy, $E_{\text {th }}^{\max }$, contained in the plasma that is heated by non-thermal electrons using the following formula:

$E_{\mathrm{th}}^{\max }=3 \varepsilon k_{\mathrm{B}} T n_{\mathrm{e}}^{-1}$ ergs

where $k_{\mathrm{B}}$ is the Boltzmann constant, $\varepsilon$ is the emission measure, $T$ is the temperature and $n_{\mathrm{e}}$ is the electron density of the plasma. Values were obtained for the maximum of the impulsive SXR brightening.

The obtained values of $E_{\mathrm{nth}}$ and $E_{\mathrm{th}}^{\mathrm{max}}$ are presented in Table 2 and plotted in Fig. 12. A correlation is seen, however the correlation coefficient is lower than for the plots of the HXR versus SXR responses (Fig 9). The higher scatter in Fig. 12 is due to uncertainties in the estimation of some parameters, especially the cut-off energy, $E_{0}$, and the volume of SXR emission needed to obtain the electron density, $n_{\mathrm{e}}$.

We know the electron density reasonably well (we estimate the typical relative error to be of the order of $20 \%-30 \%$ ), but the four broad-band channels of the HXT are not sufficient for a reasonable estimate of the cut-off energy, $E_{0}$. We arbitrarily choose a value of $14 \mathrm{keV}$.

For an independent estimation of the cut-off energy, $E_{0}$, we used Eq. (2). For each event we chose the value of the cutoff energy, $E_{0}$, to balance the value of the energy deposited by the non-thermal electrons, $E_{\mathrm{nth}}$, and the value of the maximum thermal energy, $E_{\mathrm{th}}^{\max }$. The majority of the obtained values of $E_{0}$ (Table 2) fall within the interval of 10-22 keV. This confirms the idea that the cut-off energy may vary from flare to flare and may even vary during a given flare. We stress the relatively lowenergy location of the cut-off energy (e.g. Farnik 1997) which supports the importance of low-energy electrons in the energy balance of the impulsive phase.

We compared the power-law index, $\gamma$, of the HXR photon flux spectrum with some other characteristics of the impulsive SXR brightening corresponding to the HXR burst (e.g. the SXR/HXR delay, and the $t / t_{\mathrm{HXR}}$ ratio). However, no clear correlation has been found.

\section{Conclusions}

In this paper we analyzed the impulsive phase of 46 solar flares for which impulsive SXR brightenings were clearly seen. Flares listed in Table 1 show different levels of magnetic complexity, a wide range of released energy and varying position on the solar disk. Nevertheless, the characteristics recorded for impulsive SXR brightenings are relatively uniform due to the physical conditions needed for the generation of the brightenings. Impulsive SXR brightenings are due to thermal radiation 
of plasma situated at the entrance of magnetic coronal structures into the denser atmosphere. This plasma is heated by nonthermal electron beams accelerated somewhere in the corona.

On the basis of the presented histograms (Figs. 6-8) we can describe the properties of something like a "typical" impulsive SXR brightening. It lasts about $0.5-1 \mathrm{~min}$. and has a quasisymmetrical time profile. The FWHM diameter of the region of the SXR response is about $2^{\prime \prime}-8^{\prime \prime}$. Its temperature is below $10 \mathrm{MK}$ and its electron density is the highest in the whole flaring structure. The pixels showing the impulsive SXR brightening are often the brightest in the flaring structure during the impulsive phase, however their total contribution to the SXR emission of the whole structure is rarely above $30 \%$. The impulsive SXR brightening shows a delay in comparison with the HXR burst and lasts several times longer. However, we have not found a reason for the inertia difference of chromospheric response to non-thermal electron beams.

The described characteristics should help to distinguish the impulsive SXR brightening from other morphological features. Particularly, any event resembling the impulsive SXR brightening for which observational characteristics are evidently different from those described in Sect. 4.1 should be interpreted with special caution. For example, a relatively low brightness of individual footpoints raises doubts about the interpretation of the 27 September 1993 flare as being an extra-strong impulsive SXR brightening (Harra-Murnion et al. 1997). In our opinion this event is a hybrid flare. The collected characteristics should be useful also for theoretical modeling of the impulsive phase of flares.

A generally good spatial and temporal correlation between impulsive SXR brightenings and HXR bursts strongly suggests the same origin - non-thermal electron beams. For this reason the SXT images significantly complement the HXT images in the monitoring of the non-thermal electron precipitation during the impulsive phase of the flare. The role of the SXT in such investigations is very important because it offers better spatial resolution and dynamical range than the HXT.

In many investigated flares we observe that the individual footpoint shows a response to non-thermal electrons at different moments of time (e.g. see Fig. 4). It is difficult to explain such a difference considering the time of propagation of nonthermal electrons or the inertia of chromospheric response. The time difference of footpoint reactions can be explained by the turbulent kernel model (Jakimiec et al. 1998; Jakimiec 2002). In this model non-thermal electrons are able to escape outside the turbulent kernel when a reconnection of kernel lines with external magnetic lines of force occurs. If the external lines are rooted somewhere in the chromosphere we observe the SXR and HXR response there.

For 37 events we were able to make a quantitative comparison of the impulsive response in flare footpoints observed in the SXR and the HXR images. During the impulsive phase a directly heated plasma emitting the SXRs accounts for additional contribution from the flare footpoints. The time profile of its SXR emission resembles the HXR light curve. Therefore, the strong correlation between the SXR and HXR response (Fig. 9) can be considered a proof that impulsive SXR brightenings are directly responsible for the Neupert effect. We also found

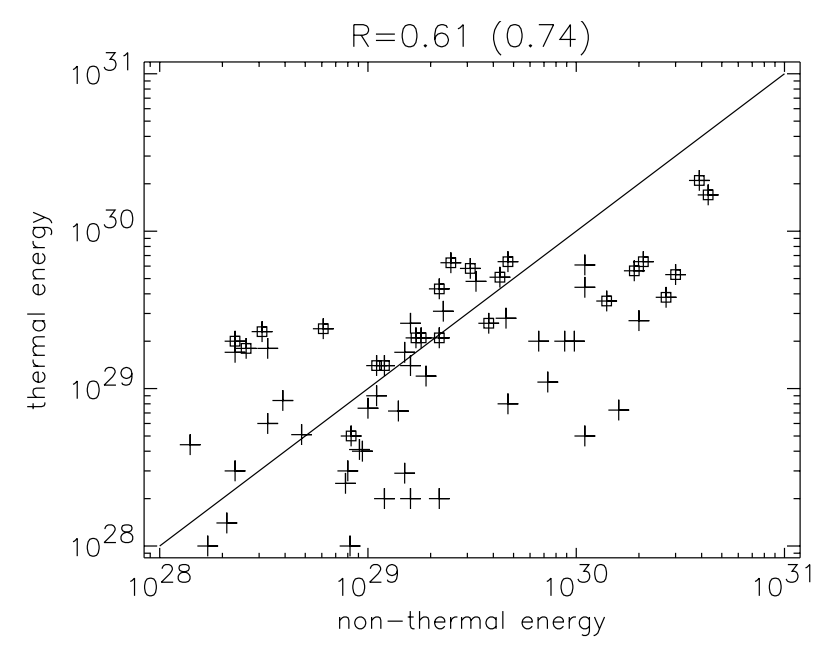

Fig. 12. The correlation between the maximum thermal energy contained in a plasma and the total, time-integrated energy deposited by non-thermal electrons. Complete events are marked by squares. The straight line gives the location of equality of the two types of energy. Values of the correlation coefficient for all events and for complete events (in parentheses) are given at the top.

a correlation between the energies: the total, time-integrated energy deposited by non-thermal electrons and the maximum thermal energy contained in the plasma heated by those particles (Fig. 12).

We obtained some observational signatures which describe how the chromospheric evaporation does occur. First, we measure the velocities of plasma motions due to the chromospheric evaporation directly from the SXT images (Tomczak 1997). This is an additional method to investigate this parameter. Especially important is that the developed method complements the classical method - the measure of blueshifts in the SXR spectra - for the flares that occurred close to the solar limb. The obtained range of values, $150-700 \mathrm{~km} \mathrm{~s}^{-1}$, is similar to those estimated from the spectral measurements (e.g. Bentley et al. 1994).

Second, we confirm the result of Tomczak (1999) that a steeper energy spectrum of HXR photons (higher power-law index) causes a higher relative productivity of SXRs. This result, together with the low-energy cut-off estimation for the HXR photon spectra, suggests that the chromospheric evaporation is caused mainly by relatively low-energy electrons. Such electrons are more frequent than high-energy ones. Moreover, more energetic electrons should reach denser atmospheric layers (Farnik et al. 1997) where they produce an impulsive reaction seen in the UV radiation. A higher radiation loss in this waveband limits the contribution of the denser layers to the chromospheric evaporation.

Such a scenario may be examined by using simultaneously the Yohkoh, the SOHO/EIT and the TRACE images. The higher quality of RHESSI data makes it possible to have a better insight into the non-thermal electron precipitation. Further analysis is in progress.

Acknowledgements. The Yohkoh satellite is a project of the Institute of Space and Astronautical Science of Japan. We acknowledge many 
useful comments and discussions of Professor J. Jakimiec. We thank also the referee, Dr. S. W. McIntosh, for valuable remarks which led to substantial improvement of this paper. This investigation has been supported by grant No. 2 P03D 00123 from the Polish Committee for Scientific Research (KBN)

\section{References}

Alexander, D., \& Metcalf, T. R. 1997, ApJ, 489, 442

Antonucci, E., Dodero, M. A., Martin, R., et al. 1993, ApJ, 413, 786

Bentley, R. D., Doschek, G. A., Simnett, G. M., et al. 1994, ApJ, 421, L55

Brown, J. C. 1971, Sol. Phys., 18, 489

Crosby, N. B., Aschwanden, M. J., \& Denis, B. R. 1993, Sol. Phys., 143,275

Dennis, B. R., \& Zarro, D. M. 1993, Sol. Phys., 146, 177

Farnik, F., Hudson, H. S., \& Watanabe, T. 1997, A\&A, 320, 620

Hara, H., Tsuneta, S., Lemen, J. R., Acton, L. W., \& McTiernan, J. M. 1992, Publ. Astron. Soc. Jpn, 44, L135

Harra-Murnion, L. K., Culhane, J. L., Hudson, H. S., et al. 1997, Sol. Phys., 171, 103

Hori, K., Yokoyama, T., Kosugi, T., \& Shibata, K. 1998, ApJ, 500, 492

Hudson, H. S., Strong, K. T., Dennis, B. R., et al. 1994, ApJ, 422, L25
Jakimiec, J. 2002, ESA-SP 506, 645

Jakimiec, J., Tomczak, M., Falewicz, R., Phillips, K. J. H., \& Fludra, A. 1998, A\&A, 334, 1112

Kosugi, T., Makishima, K., Murakami, T., et al. 1991, Sol. Phys., 136, 17

Lee, T. T., Petrosian, V., \& McTiernan, J. M. 1995, ApJ, 448, 915

Martens, P. C., Acton, L. W., \& Lemen, J. R. 1995, Sol. Phys., 157, 141

Metcalf, T. R., Hudson, H. S., Kosugi, T., Puetter, R. C., \& Piña, R. K. 1996, ApJ, 466, 585

McTiernan, J. M., Fisher, G. H., \& Li, P. 1999, ApJ, 514, 472

Morrison, M., Yohkoh Analysis Guide, Lockheed Palo Alto Res. Lab. Rep. LMCSC-P098510, 1994

Ogawara, Y., Takano, T., Kato, T., et al. 1991, Sol. Phys., 136, 1

Sakao, T. 1994, Ph.D. Thesis, University of Tokyo

Sato, J., Kosugi, T., \& Makishima, K. 1999, Publ. Astron. Soc. Jpn, 51,127

Spitzer, L. 1962, Physics of Fully Ionizated Gases (New York: Interscience)

Tomczak, M. 1997, A\&A, 317, 223

Tomczak, M. 1999, A\&A, 342, 583

Tsuneta, S., Acton, L., Bruner, M., et al. 1991, Sol. Phys., 136, 37

Veronig, A., Vršnak, B., Dennis, B. R., et al. 2002, A\&A, 392, 699 